\title{
Investigating the Transferability of Calibrated Microsimulation Parameters for Operational Performance Analysis in Roundabouts
}

\author{
Vincenzo Gallelli, Teresa Iuele, Rosolino Vaiana, and Alessandro Vitale \\ Department of Civil Engineering, University of Calabria, Rende, 87036 Cosenza, Italy \\ Correspondence should be addressed to Vincenzo Gallelli; vincenzo.gallelli@unical.it
}

Received 31 July 2016; Revised 17 December 2016; Accepted 4 January 2017; Published 19 January 2017

Academic Editor: Dongjoo Park

Copyright (C) 2017 Vincenzo Gallelli et al. This is an open access article distributed under the Creative Commons Attribution License, which permits unrestricted use, distribution, and reproduction in any medium, provided the original work is properly cited.

\begin{abstract}
Microsimulation models are widespread for the analysis of roundabouts operational performance providing realistic modelling of vehicle movements. These models are based on many independent parameters to describe traffic and driver behaviour, which need to be calibrated in order to better match field data. In practice, despite the well-recognized importance of calibration and validation processes, simulation is conducted under default values because of difficulties in field data collection and deficiency in available guidelines. These issues can be faced by using transferability methodologies that allow applying the parameters calibrated for a case study to other similar locations. Therefore, this paper investigates the suitability of the transferability procedure adopting both the application-based and estimation-based approaches, by considering two roundabouts and a microsimulation tool. A Genetic Algorithm technique was used to determine the best estimates of these model parameters. After that, the authors compared fieldmeasured with simulated queue lengths, considering four different scenarios. The results show that the application of Wiedemann 99 parameters calibrated for the first case study to the second one allows reducing the RMSNE more than 50\%, thus confirming an acceptable level of transferability of these parameters between the two case studies.
\end{abstract}

\section{Introduction and Background}

In the last few decades, simulation models have been widespread for transport system analysis and management, also in relation to traffic safety, and to evaluate a range of new ITS (Intelligent Transport System) applications [1]. In fact, simulation is less expensive, safer, and faster when compared with field implementation and testing [2]. In particular, as regards roundabout analysis, microsimulation models allow a dynamic approach to be used in order to predict intersection operational performance (capacity, levels of service, stop-line delay, etc.) and operating speed profiles [3]. Furthermore, they provide other advantages, such as realistic modelling of vehicle movements, also in relation to roundabout geometric features [4], the possibility of analysing queues spatial distributions, and more refined estimations of emissions and fuel consumption [5].

Nowadays, many commercial microsimulation tools have been developed and become widespread. AIMSUN [6],
VISSIM [7], and PARAMICS [8] are the most common of them, together with SimTraffic and Corsim.

Microscopic simulation models are based on modelling of vehicle kinematics and interactions; movements are governed by gap acceptance, car following, lane-changing, and other models and are typically calculated for each vehicle at every specified time-step. In fact, they use an interval-based simulation to describe traffic operations [9].

The usability of each tool in terms of both qualitative (applicability, ease, sensitivity to geometry, etc.) and quantitative aspects (measures of operational performance, such as delays, queue length, and capacity) has to be analysed in order to select the most adequate model for simulation [10].

Microscopic simulation models are characterized by a flexible and user-definable methodology to evaluate traffic operations; moreover, the models are based on many independent parameters that are used to describe traffic flow and driver behaviour. Nevertheless, the default values provided for each parameter need to be changed by users 
in order to better match real field conditions through an adequate calibration and validation process [11, 12]. Using default calibration parameters, in fact, can produce unreliable simulation results. Both calibration and validation procedures enhance model accuracy, minimizing the discrepancy between model results and measurements or observations [13].

As stated above, several software packages for simulating traffic have been developed and used to predict the actual operational performance of roundabouts. Therefore, a proper calibration of the simulation parameters is still a key issue which must be faced.

In the study conducted by Gagnon et al. [14], the calibration potential of two analytical models (aaSIDRA and RODEL) and three microsimulation tools (PARAMICS, SimTraffic, and VISSIM) was investigated. In relation to the selected case studies (two modern roundabouts in New England), the authors found that VISSIM was the most versatile software for calibration purposes; on the contrary, RODEL seemed to be the least one and PARAMICS was quite insensitive to changes in input parameters for calibration. Results in terms of average delays also showed that, after calibration, the average percentage error was reduced from $52 \%$ to $13 \%$ and from $68 \%$ to $0.3 \%$ by using aaSIDRA and VISSIM models, respectively. Nevertheless, the authors highlighted that these findings were site specific and, therefore, the previous results needed to be validated on other similar test sites.

Chen and Lee [15] investigated the accuracy of three software packages (RODEL, SIDRA, and VISSIM), which offer different calibration capabilities, in predicting multilane roundabout capacity and delays by making a comparison between uncalibrated results and field-measured data. As regards capacity estimates (at different circulating flow rates), results highlighted that RODEL slightly overestimated capacity at each entry, whereas SIDRA uncalibrated capacity was noticeably higher than collected field data for two of the three approaches. For the third approach, instead, results were closer to the measured data probably because this software performed better under high-demand conditions. Finally, VISSIM provided good capacity estimates.

The importance of calibration in terms of comparability between field-measured data and simulated results was also highlighted by [16]. In this paper, the validation of the calibrated VISSIM model (by neural network approach) was performed on one-lane roundabouts and it was carried out in two steps. The first one, with a set of data collected at the same roundabout, is where the calibration was done; the second phase, instead, was performed on the measured data related to a second roundabout. Three traffic parameters such as traveling time, maximum queue length, and number of vehicles stopping at the entrance of the roundabout were investigated. The results of the first validation showed that the uncalibrated model provided a maximum queue length lower than the measured value of about $28 \%$, whereas the calibration process allowed reduction of the percentage difference between the estimates of all parameters and the measured data to about $5 \%$. The results of the second validation step showed that the model provided traveling time values similar to real data, whereas the estimates of the maximum queue length and the number of stopping were $26 \%$ greater and around $11 \%$ lower than the field measurements, respectively. The calibration procedure reduced these gaps to a difference between measured and simulated parameters lower than $7 \%$.

A trial and error approach for the calibration of two double lane roundabouts of different diameter was used in the research carried out by [17]. In this paper the difference between observed field data and simulated values for entry flows was expressed in terms of Mean Absolute Percentage Error (MAPE). The best configuration of the input parameter was selected and, for validating the model, measured speeds at the weaving section between leg 1 and leg 2 of both roundabouts were compared with the weaving speeds obtained from VISSIM. The MAPE values obtained from this comparison were found as 12.35 and 13.13 for the first and the second roundabout, respectively. Furthermore, a comparison between entry flows and circulating flows values obtained from VISSIM microsimulation and field data was carried out for both the roundabouts. Also, in this case, the results highlighted a strict correlation between estimates and real values. Finally, the authors investigated the capacity results, showing that simulated capacities were the closest to the observed ones if compared to the results obtained from the application of other methodologies (such as HCM and German models, IRC method, and TRRL regression method). Differences from a minimum of $7 \%$ to a maximum of $19 \%$ (according to the leg analysed of the roundabout) were found between VISSIM results and observed capacity values.

Unfortunately, the calibration process is often neglected because of difficulties in field data collection, lack of sufficient data, and/or deficiency in available procedure guidelines [18]. These limitations can be avoided by transferring the parameters calibrated for a case study to other locations that are comparable in geometry and traffic conditions. However, the transferability of calibrated microsimulation parameters at different contexts must be investigated [19]. For this reason, recent researches have been oriented to the use of automatic calibration for traffic simulation models because of the increase in the number of parameters to manage. Therefore, the calibration process is considered as an optimization problem. For microscopic models, sensitivity analysis and a trial and error method can be used for automatic calibration, as shown in $[2,20]$; however these two procedures are very time-consuming. Other studies, instead, focus on the calibration of input parameters by genetic algorithm (GA) [21], neural networks [16], or multistart algorithm [22].

In this regard, the MULTITUDE [23] Project (Methods and tools for supporting the Use, caLibration, and validaTIon of Traffic simUlations moDEls), strongly supported by the European Commission through the Joint Research Centre, plays a key role. It was an Action (TU0903) supported by the EU COST office (European Cooperation in Science and Technology) and focused on the issue of uncertainty in traffic simulation and of calibration and validation as tools to manage it. In this document, the 4 Working Groups assessed the current situation regarding guidelines for traffic simulation model calibration and validation worldwide and suggested potential ways in which they can be addressed. 
Starting from an extensive review of existing guidelines of the application of traffic microsimulation models, MULTITUDE addressed thirteen key issues that are faced in performing traffic simulation projects, such as sensitivity analysis, appropriate definitions of calibration and validation, calibration methodologies, effect of parameters (and different types of parameters) on output, and indicators to use for calibration and validation assessment. In conclusion, the review highlighted that the production of new guidelines in this area was something for which there was a clear need.

In [20] the authors instead adopted a metamodel-based technique for model sensitivity analysis and applied it to the AIMSUN mesoscopic model. Considering that the main obstacle to an extensive use of the most sophisticated techniques is the high number of model runs, in this study, a sensitivity analysis was tested not on a model but on its metamodel approximation. Sobol sequences and Gaussian process metamodels were recognized as the appropriate choices. The proposed methodology was assessed by comparing the results of the application of variance-based sensitivity analysis techniques with the simulation model and with a metamodel estimated with 512 model runs for a variety of traffic scenarios and model outputs. The results confirmed the power of the proposed methodology.

\section{Organization and Scope}

The objective of this paper is to evaluate the transferability of microsimulation model parameters (VISSIM) between two different roundabouts comparable in traffic conditions and which belong to the class of Conventional Roundabouts, according to the Italian Standard [24]. In particular, considering the operational performance of the intersections, the aim is to determine, in the two contexts, how the transferred parameters affect results in terms of distance between fieldmeasured and simulated queue lengths.

The paper is organized according to the following structure:

(i) the first section provides an outline of the state of the art of the use of microsimulation for determining the operational performance of road infrastructures and the different calibration techniques;

(ii) in the second section, the focus is on the description of the case studies followed by the data collection experiment and the microsimulation tool description;

(iii) after that, the paper deals with the calibration methodology used for a case study, followed by the transferability methodology of the calibrate parameters to the other case study;

(iv) finally, the paper concludes with a summary of results and a discussion of the implications for future researches.

\section{Case Study}

3.1. Marconi Roundabout. The first case study roundabout is placed in Cosenza, South Italy. It is an important at-grade

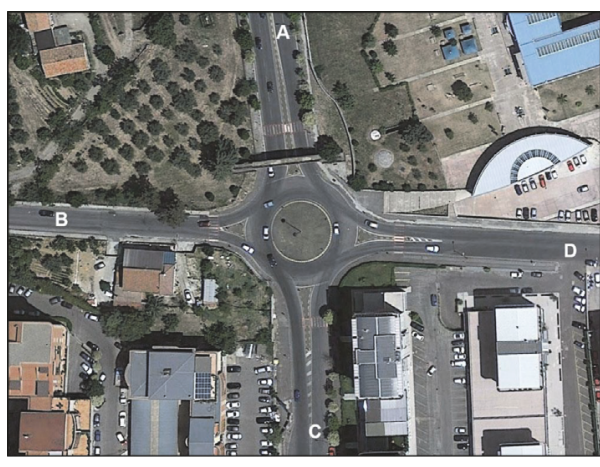

Figure 1: Aerial image of Marconi Roundabout.

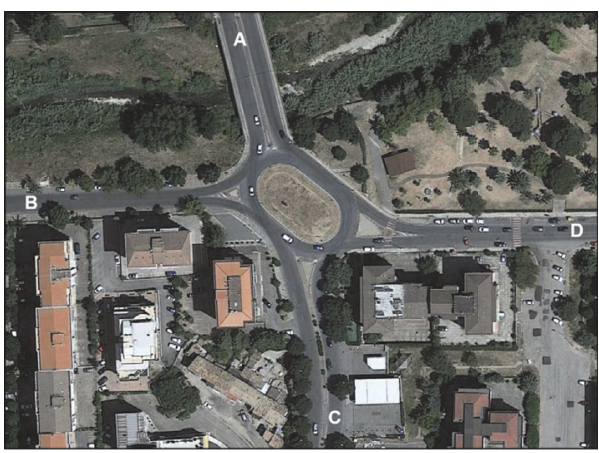

Figure 2: Aerial image of Cosmai Roundabout.

intersection because of its position along a strategic busy road. It is a roundabout with four entries equally spaced between them and with an inscribed circle diameter of 38.00 meters. It presents two main approaching branches (legs A and $C$ in Figure 1), both with two entry lanes, and two secondary ones (legs B and D in Figure 1), instead, with a single entry lane.

3.2. Cosmai Roundabout. The second roundabout is also located in the city of Cosenza. This intersection cuts off a major four-lane divided arterial road (legs $\mathrm{A}$ and $\mathrm{C}$ in Figure 2) and a minor two-lane undivided road (legs $B$ and $\mathrm{D}$ in Figure 2). The roundabout presents an elliptical ring (without lane marking on the circle) with two external diameters of 38.00 and 55.00 meters and with four branches at different angles.

\section{Data Collection}

4.1. Video Image Processing Technique. For both case studies, a video image processing technique was used to acquire vehicle data for the calibration of the microsimulation package (VISSIM). The video analysis was performed with Adobe Premiere Software, on a frame by frame basis. The temporal distance between each frame was set up to $1 / 25$ of a second. The authors used a differential GPS instrument in order to position virtual detectors on each roundabout; this system ensures a positioning error of 10 centimeters (Figure 3). Knowing the position (latitude and longitude) of three reference points and the camera location, about 


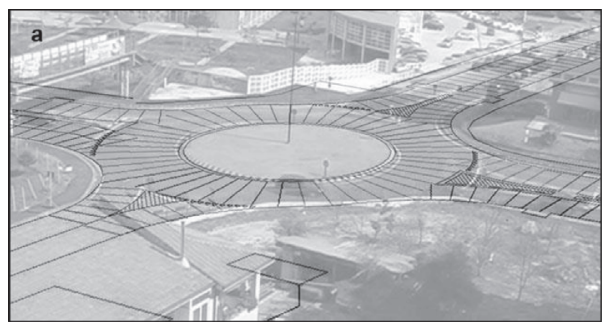

(a)

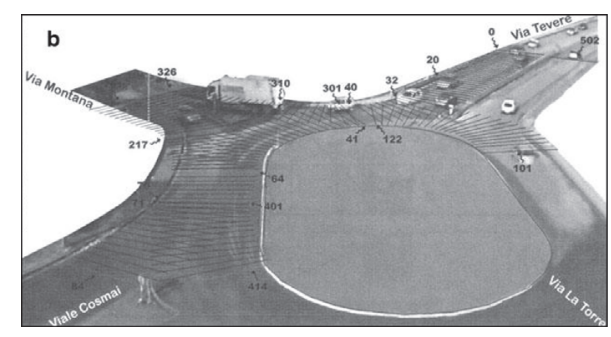

(b)

FIgURE 3: Position of virtual detectors used for data acquiring.

TABLE 1: O/D matrix for Marconi Roundabout.

\begin{tabular}{lcccc}
\hline veic/h & A & B & C & D \\
\hline A & 0 & 151 & 1080 & 144 \\
B & 104 & 0 & 153 & 94 \\
C & 472 & 75 & 0 & 147 \\
D & 72 & 135 & 194 & 0 \\
\hline
\end{tabular}

109 (Marconi Roundabout, Figure 3(a)) and 176 (Cosmai Roundabout, Figure 3(b)) virtual detectors were positioned on video, matching accurately vehicle progression along each trajectory [25].

4.2. Traffic Flows, Speed Distributions, and Queue Lengths. For Marconi Roundabout (Figure 3(a)), traffic parameters were recorded during a typical weekday between 11.30 a.m. and 12.30 p.m. Traffic flows, queue lengths, approaching delays, time of services, critical gaps and speed distributions (at the entries and along the ring) were carried out. In particular, Table 1 shows the O/D matrix, homogenized in vehicle per hour, while Table 2 reports the queue lengths for each entry.

Considering Cosmai Roundabout (Figure 3(b)), traffic data were also obtained during a typical weekday between 11.30 a.m. and 12.30 p.m. Traffic flows are reported in Table 3. In order to determine vehicles' speed distribution, individual vehicle speed profiles were obtained from video capture. The speed profiles were found to be normally distributed with mean. The average and the maximum queue lengths for each entry, instead, are reported in Table 4.

\section{Microsimulation Tool}

Roundabouts microsimulation often shows many complexities, because it is not easy to define all the geometric and user-behavioural features. In this paper, the authors have used VISSIM, which is a widespread microscopic simulation model. It is a time-step and behavioural model developed to simulate traffic. It is based on psychophysical car following model and a rule-based algorithm for lateral movements realized by Wiedemann [26] which assumes that drivers can have one of four driving modes: free driving, approaching, following, and braking [7].

In this study, the VISSIM model was developed accurately to match real field conditions. First, the geometry of the
TABLE 2: Queue lengths measured for each entry of Marconi Roundabout.

\begin{tabular}{lcccc}
\hline & A & B & C & D \\
\hline Average queue length $(\mathrm{m})$ & 36.6 & 17.7 & 7.4 & 7.1 \\
Max queue length $(\mathrm{m})$ & 96.0 & 54.0 & 18.0 & 30.0 \\
\hline
\end{tabular}

TABle 3: O/D matrix for Cosmai Roundabout.

\begin{tabular}{lcccc}
\hline veic/h & A & B & C & D \\
\hline A & 0 & 176 & 702 & 294 \\
B & 374 & 0 & 82 & 112 \\
C & 456 & 88 & 36 & 34 \\
D & 510 & 175 & 46 & 2 \\
\hline
\end{tabular}

TABLE 4: Queue lengths measured for each entry of Cosmai Roundabout.

\begin{tabular}{lcccc}
\hline & A & B & C & D \\
\hline Average queue length $(\mathrm{m})$ & 31.8 & 19.4 & 5.5 & 9.3 \\
Max queue length $(\mathrm{m})$ & 89.0 & 63.0 & 16.00 & 38.00 \\
\hline
\end{tabular}

intersection (e.g., number of lanes, lanes widths, and turning radius) was extracted from an aerial photo and then drawn in a technical drawing. After that, links and connectors were set up with the real dimensions. Furthermore, there are three principal features, which are very important to set for a correct simulation [27]: (a) the speed distributions for approaches, reduced speed zones, and circulatory carriageway; (b) the priority rules; and (c) the traffic assignment.

The results were recorded by using average queue lengths and the relative values of standard deviations.

\section{Calibration Procedure}

Two different calibration processes were carried out using data from both considered roundabouts (Marconi Roundabout and Cosmai Roundabout). In order to study the existing correlation between the simulated and the fieldmeasured operational performance of the roundabouts, the authors proposed a calibration procedure characterized by a multistage methodology. The first stage of the process involves the macroscopic variables of traffic flow. In particular, at this step, observed traffic volumes and speed distribution were considered in input to microsimulation. 
TABLE 5: Wiedemann 99 input parameters' low and high values and their short description.

\begin{tabular}{|c|c|c|c|c|}
\hline Factor & Parameter & Low level $(-1)$ & High level (+1) & Description \\
\hline A & Desired Speed (average) $(\mathrm{Km} / \mathrm{h})$ & 20 & 50 & Individual free flow speed. \\
\hline B & Desired Speed (standard deviation) & 4 & 7 & $\begin{array}{l}\text { Standard deviation of the } \\
\text { desired speed. }\end{array}$ \\
\hline $\mathrm{C}$ & Desired Deceleration $\left(\mathrm{m} / \mathrm{s}^{2}\right)$ & -4.5 & -1.3 & $\begin{array}{l}\text { The maximum deceleration } \\
\text { drivers are willing to apply } \\
\text { in "normal" condition. }\end{array}$ \\
\hline $\mathrm{D}$ & Observed vehicles ahead & 1 & 4 & $\begin{array}{l}\text { Influences drivers' ability to } \\
\text { adjust their speed/distance } \\
\text { according to a given } \\
\text { number of lead vehicles. }\end{array}$ \\
\hline $\mathrm{E}$ & $\mathrm{CC} 0$ & 0.5 & 3 & $\begin{array}{l}\text { Standstill distance }(\mathrm{m}) \text {, } \\
\text { defines the desired distance } \\
\text { between stopped cars. }\end{array}$ \\
\hline $\mathrm{F}$ & $\mathrm{CC} 1$ & 0.5 & 1.5 & Headway time (s). \\
\hline G & CC2 & 1,5 & 6 & Following variation $(\mathrm{m})$ \\
\hline $\mathrm{H}$ & CC3 & -15 & -4 & $\begin{array}{l}\text { Threshold for the entering } \\
\text { "following." }\end{array}$ \\
\hline I & CC5 & 0.1 & 2 & $\begin{array}{l}\text { Positive "following" } \\
\text { threshold. }\end{array}$ \\
\hline $\mathrm{J}$ & CC6 & 2 & 20 & $\begin{array}{l}\text { Speed dependency of } \\
\text { oscillation. }\end{array}$ \\
\hline $\mathrm{K}$ & CC7 & 0.1 & 0.3 & Oscillation acceleration. \\
\hline $\mathrm{L}$ & CC8 & 0.5 & 3.5 & Standstill acceleration. \\
\hline
\end{tabular}

The second calibration step is centered on microscopic parameters regulating VISSIM car following behaviour of the drivers. In order to enhance the correlation between fieldmeasured and simulated queue lengths, the Wiedemann 99 car following input parameters were calibrated.

The objective is to find the configuration of VISSIM car following parameters (Wiedemann 99 car following model) that ensures a good level of correlation between field-measured and simulated queue lengths. At this stage, the calibration procedure is based on a statistical screening of inputs (Plackett-Burman with fold-over), establishing a linear expression relating significant parameters to queue length. Subsequently, the best estimates of this model parameters are determined using a genetic algorithm technique $[28,29]$.

\section{Calibration Results}

Traffic volume and vehicle speed distribution were set up according to observed values. On the basis of vehicle interactions in roundabout traffic stream, twelve parameters of the Wiedemann 99 car following model were chosen for the second calibration stage. A short description of the parameters, together with their corresponding low/high values [2, 28-30], is reported in Table 5. Further details on the Wiedemann 99 parameters are reported in the VISSIM user manual [7].

In order to establish the level of interaction of the twelve parameters considered, the Plackett-Burman with fold-over design was applied [31], performing the generation of 36 different parameters combinations with three replicates for each case study (a total of 108 combinations). The
TABLE 6: Significance of factors according to ANOVA test.

\begin{tabular}{lccc}
\hline Factor & Parameter & $T$ & $p$ \\
\hline Constant & & 57.73 & 0.000 \\
A & Desired Speed (average) $(\mathrm{Km} / \mathrm{h})$ & -2.30 & 0.023 \\
B & Desired Speed (standard deviation) & -0.13 & 0.894 \\
C & Desired Deceleration $\left(\mathrm{m} / \mathrm{s}^{2}\right)$ & -0.87 & 0.388 \\
D & Observed vehicles ahead & 1.96 & 0.053 \\
E & CC0 & 10.01 & 0.000 \\
F & CC1 & 9.87 & 0.000 \\
G & CC2 & 7.90 & 0.000 \\
H & CC3 & -2.78 & 0.007 \\
I & CC5 & -1.42 & 0.159 \\
J & CC6 & 2.31 & 0.023 \\
K & CC7 & 3.66 & 0.000 \\
L & CC8 & -11.35 & 0.000 \\
\hline
\end{tabular}

Plackett-Burman with fold-over fractional design represents a particular case of 2-level factorial design, particularly recommended for screening purposes. The experiment determines the statistical significance of the main interaction terms. A set of ten simulations for each combination was carried out (1080 simulations). The replicates of a single parameter combination were necessary to estimate the error due to the random nature of the simulation (each parameters' combination was simulated 30 times).

The significance at $5 \%$ level of each factor was established using ANOVA (Table 6). Table 6 highlights that Desired Speed (standard deviation), Desired Deceleration, and CC5 
(positive "following" threshold) are not statistically significant as regards the queue lengths in roundabout. The standard deviation of Desired Speed is probably not significant due to the low-speed variability. Furthermore the low-speed regime influences also the lack of significance of Desired Deceleration. In VISSIM, Desired Deceleration assumes the maximum among the following parameters: the deceleration consequence of a Desired Speed approach; the deceleration in Stop \& Go traffic situations; the deceleration consequence of an emergency stop condition; the deceleration in case of cooperative braking [7]. Considering the positive "following" threshold (CC5), it represents the threshold, which controls the speed differences during the "following" state [7]. The lack of significance is probably determined by the driving mode at the roundabout entries which is in "following" mode, according to VISSIM.

As an outcome of factorial analysis, a linear model for each considered roundabout was determined. The response variable (queue length) was estimated as a function of significant factors and their effects. For Marconi Roundabout the expression is

\section{QUEUE LENGTH (Marconi)}

$$
\begin{aligned}
= & -77.5081+0.8754 *(\text { Desired Speed })+7.4411 \\
& *(\text { Observed vehicles ahead })+45.6818 * \text { CC } 0 \\
& +112.5230 * \text { CC } 1+20.0168 * \text { CC } 2-2.8794 \\
& * \text { CC } 3+1.4665 * \text { CC } 6+208.9230 * \text { CC } 7 \\
& -43.1549 * \text { CC } 8
\end{aligned}
$$

Considering Cosmai Roundabout, the formulation of the linear equation is

\section{QUEUE LENGTH (Cosmai)}

$$
\begin{aligned}
= & -77.5981+0.8763 *(\text { Desired Speed })+7.4312 \\
& *(\text { Observed vehicles ahead })+45.6754 * \text { CC } 0 \\
& +113.5425 * \text { CC } 1+19.9874 * \text { CC } 2-2.8886 \\
& * \text { CC } 3+1.4698 * \text { CC } 6+209.2635 * \text { CC } 7 \\
& -42.9621 * \text { CC } 8
\end{aligned}
$$

The expressions (1) and (2) obtained from the previous step have an infinite number of solutions. The best estimate values of the parameters in linear equation models were obtained applying a generational genetic algorithm (GA). Typically, a genetic algorithm requires the specification of two constraints which are the genetic representation of the solution domain and the fitness function to evaluate the solution domain. For this experiment, the genetic representations of queue length, for each considered roundabout, are linear equations (1) and (2). The fitness function, which determines the precision of
TABLE 7: Best estimate of factors obtained from the generational genetic algorithm.

\begin{tabular}{lcc}
\hline Parameters & $\begin{array}{c}\text { Marconi roundabout } \\
\text { Values }\end{array}$ & $\begin{array}{c}\text { Cosmai roundabout } \\
\text { Values }\end{array}$ \\
\hline $\begin{array}{l}\text { Desired Speed } \\
\text { (average) }(\mathrm{Km} / \mathrm{h})\end{array}$ & 20.01 & 26.42 \\
$\begin{array}{l}\text { Observed vehicles } \\
\text { ahead }\end{array}$ & 4 & 4 \\
CC0 & 0.50 & 0.53 \\
CC1 & 0.52 & 0.55 \\
CC2 & 2.21 & 2.27 \\
CC3 & -7.06 & -7.15 \\
CC6 & 8.01 & 8.29 \\
CC7 & 0.27 & 0.28 \\
CC8 & 3.48 & 3.46 \\
\hline
\end{tabular}

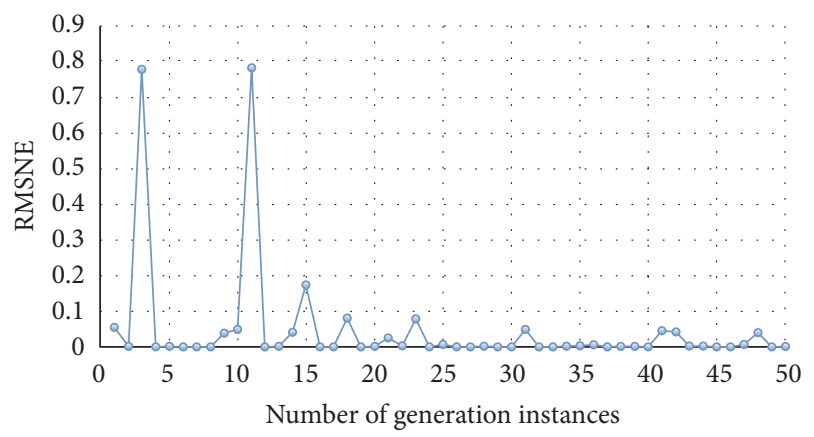

FIgURE 4: Values of the RMSNE during the optimization process for the Marconi Roundabout.

the solution, in both cases, is defined as the Root Mean Squared Normalized Error (RMSNE) [32].

$$
\mathrm{RMSNE}=\sqrt{\frac{1}{N} \sum_{i=1}^{N}\left(\frac{x_{i}-y_{i}}{y_{i}}\right)^{2}},
$$

where $x_{i}$ is simulated measurements of queue lengths; $y_{i}$ is observed measurements of queue lengths; $N$ is number of simulations.

In the first step, GA proceeded to initialize a population of solutions randomly. At each iteration, a new population was created from the old one by using the selection, crossover, and mutation operators. After 50 generation instances, the optimum solution for each case study corresponded to the parameters values reported in Table 7 .

Figure 4 shows the variability of the RMNSE during the 50 generation instances of the GA for the Marconi Roundabout calibration. Moreover it highlights that the optimum solution found is not a local minimum but the absolute minimum: the optimum value is $8.31 * 10^{-7}$ obtained at the 39 th generation.

\section{Microsimulation Parameters Transferability}

In the literature, according to $[23,33]$, there are two main approaches to assess the transferability of a certain model: 
TABLE 8: Scenario parameters for transferability investigation.

\begin{tabular}{lcccccccccccc}
\hline Scenario & $\begin{array}{c}\text { Desired Speed } \\
\text { (average) } \\
(\text { Km/h) }\end{array}$ & $\begin{array}{c}\text { Observed } \\
\text { vehicle ahead }\end{array}$ & CC0 & CC1 & CC2 & CC3 & CC6 & CC7 & CC8 & Description & $\begin{array}{c}\text { Observed } \\
\text { Speed } \\
\text { Distribution }\end{array}$ \\
\hline 1 & 50.00 & 4 & 1.50 & 0.90 & 4.00 & -8.00 & 11.44 & 0.25 & 3.50 & Default & No \\
2 & 25.43 & 4 & 1.50 & 0.90 & 4.00 & -8.00 & 11.44 & 0.25 & 3.50 & Default & Yes \\
3 & 25.43 & 4 & 0.50 & 0.52 & 2.21 & -7.06 & 8.01 & 0.27 & 3.48 & Transferred & Yes \\
4 & 26.42 & 4 & 0.53 & 0.55 & 2.27 & -7.15 & 8.29 & 0.28 & 3.46 & Calibrated & Yes \\
\hline
\end{tabular}

the application-based approach and the estimation-based approach. In the application-based approach the model parameters are calibrated for one context, defined as "base context," and applied directly (without any changes) to another location. In this way it is possible to determine how the calibrated model predicts traffic phenomena in another location [34]. Differently, in the estimation-based approach, model parameters are calibrated using data from one location and recalibrated for another context, determining how the calibrated parameters values are different between the two case studies [35].

In order to avoid the influence of a random component or a lucky parameters' combination on the results of the transferability investigation methodology, both the applicationbased and the estimation-based approaches were applied. In particular, according to the first approach, the calibrated parameters obtained using data from Marconi Roundabout were applied for the simulation of Cosmai Roundabout. Furthermore, following the second approach, the calibration process was applied also using Cosmai Roundabout dataset. According to [19], the methodology was made up of four scenarios in which the correlation between field-measured and simulated queue lengths was determined (Table 8).

(i) Scenario 1. In this step Cosmai Roundabout was modelled and simulated, using VISSIM default speed distribution and the Wiedemann 99 default parameters (without any calibration).

(ii) Scenario 2. Cosmai Roundabout was simulated using observed speed distribution and Wiedemann 99 default parameters.

(iii) Scenario 3. Cosmai Roundabout was simulated using observed speed distribution and the Wiedemann 99 parameters calibrated for Marconi Roundabout, directly transferred without any recalibration process, according to the application-based approach.

(iv) Scenario 4. Considering the estimation-based approach, the Wiedemann 99 significant factors calibrated for Cosmai Roundabout were compared with the same ones calibrated for Marconi Roundabout, evaluating the degree of transferability.

For the first three scenarios, the performance of transferred parameters (validation process) is obtained by examining the Root Mean Square Normalized Error (RMSNE) value, relative to observed and simulated queue lengths. In Scenario
TABLE 9: Comparison between field-measured and simulated queue lengths in Scenario 1.

\begin{tabular}{lc}
\hline Simulated & Queue length $(m)$ \\
\hline Mean & 22.99 \\
Standard deviation & 7.91 \\
Number of simulations & 20 \\
\hline Observed & Queue length $(m)$ \\
\hline Mean & 16.50 \\
Standard deviation & 4.90 \\
\hline RMSNE & 1.29
\end{tabular}

4, according to [19], the transferability of each parameter was investigated through a comparison between the parameter values calibrated for each roundabout considered. The measure adopted is the Percentage of Change [32] represented in the following equation:

$$
\% \text { of change }=\frac{(C 1-C 2)}{\operatorname{Max}-\operatorname{Min}} * 100,
$$

where $C 1$ is the parameter value calibrated for Marconi Roundabout; $C 2$ is the parameter value calibrated for Cosmai Roundabout; Max is the maximum value of parameter; Min is the minimum value of parameter.

The minimum and the maximum parameter values are those reported in Table 5. Considering that VISSIM simulates traffic in a one-shot simulation, 20 simulations were conducted for each set of scenarios (multiple-run simulations with running time of one hour for each simulation), in order to get a statistically valid estimate of queue lengths.

8.1. Scenario 1. In this scenario, Cosmai Roundabout was simulated employing VISSIM default parameters, without a calibration stage. From Table 9 it can be seen that, comparing field-measured and simulated queue lengths, the RMSNE value of 1.29 is high. Therefore, the use of an uncalibrated microsimulation tool should be avoided.

8.2. Scenario 2. In this scenario, Cosmai Roundabout was simulated, replying observed speed distribution in VISSIM. On the contrary, the Wiedemann 99 parameters were left with their default values. Table 10 shows that, in this case, the RMSNE is 1.35 , which seems to underline the worst correlation between simulated and field-observed queue lengths when compared to Scenario 1. Probably this problem 
TABLE 10: Comparison between field-measured and simulated queue lengths in Scenario 2.

\begin{tabular}{lc}
\hline Simulated & Queue length $(\mathrm{m})$ \\
\hline Mean & 23.38 \\
Standard deviation & 8.07 \\
Number of simulations & 20 \\
\hline Observed & Queue length $(\mathrm{m})$ \\
\hline Mean & 16.50 \\
Standard deviation & 4.90 \\
\hline RMSNE & 1.35 \\
\hline
\end{tabular}

TABLE 11: Comparison between field-measured and simulated queue lengths in Scenario 3.

\begin{tabular}{lc}
\hline Simulated & Queue length \\
\hline Mean & 17.63 \\
Standard deviation & 5.99 \\
Number of simulations & 20 \\
\hline Observed & Queue length \\
\hline Mean & 16.50 \\
Standard deviation & 4.90 \\
\hline RMSNE & 0.59 \\
\hline
\end{tabular}

is due to an unrealistic driver behaviour simulated by the Wiedemann 99 car following model with default parameters values. The default configuration of the Wiedemann 99 car following model in VISSIM is not suitable for the case study considered.

8.3. Scenario 3. In this scenario, for the simulation of Cosmai Roundabout, the observed speed distribution and the Wiedemann 99 parameters calibrated for Marconi Roundabout were used. As it can be seen from Table 11, the RMSE between simulated and field-observed queue lengths halved with respect to both Scenario 1 and Scenario 2. The RMSNE value indicates an acceptable level of transferability of the Wiedemann 99 parameters between the two case studies.

8.4. Scenario 4. As reported in Table 12, applying the estimation-based approach, there is no change in parameter values calibrated for the two different contexts.

These results confirmed the transferability of overall parameters, corroborating the results of the applicationbased approach. In this way, the influence of random or lucky combinations on the transferability results was avoided. The only parameter, which showed a considerable Percentage of Change, was the Desired Speed, but this is explainable if we consider that the two roundabouts are located along two urban roads with specific difference between them. Moreover, the percentage is a little greater than $20 \%$, which is an acceptable value for this parameter according to [19].

\section{Conclusions}

In this paper, the authors investigated and evaluated the transferability of microsimulation model parameters between field-measured and simulated queue lengths at two Conventional Roundabouts under similar traffic conditions. A video image processing technique was used to acquire vehicle data for the calibration of the microsimulation package (VISSIM). In the first part of the paper, a multistage calibration procedure was carried out for the Marconi Roundabout, determining the Wiedemann 99 car following model coefficients values which gave reasonable results in terms of observed queue lengths. A genetic algorithm technique was used to determine the best estimates of these model parameters. Then, in order to demonstrate the transferability of the calibrated simulation parameters, the authors adopted both the application-based and the estimation-based approaches. In particular, according to the former, the calibrated parameters deriving from Marconi Roundabout were applied for the simulation of Cosmai Roundabout. Furthermore, following the second approach, the calibration process was applied also using Cosmai Roundabout dataset. After that the authors compared field-measured with simulated queue lengths, considering four different scenarios:

(i) Cosmai Roundabout simulation using VISSIM default speed distribution and the Wiedemann 99 default parameters (without any calibration);

(ii) Cosmai Roundabout simulation using observed speed distribution and Wiedemann 99 default parameters;

(iii) Cosmai Roundabout simulation using observed speed distribution and the Wiedemann 99 parameters calibrated for Marconi Roundabout;

(iv) Cosmai Roundabout simulation with the Wiedemann 99 significant factors calibrated for itself against Marconi Roundabout simulation with the Wiedemann 99 significant factors calibrated.

Finally, the results, evaluated in terms of RMSNE and degree of transferability between parameters calibrated for Marconi and Cosmai Roundabouts, showed that

(i) in Scenario 1, the default Wiedemann parameters give an RMSNE of 1.29;

(ii) in Scenario 2, despite the use of the observed speed distribution with Wiedemann default parameters, the RMSNE increases up to 1.35;

(iii) in Scenario 3, after the calibration, the RMSNE value decreases until 0.59 as a consequence of the use of the Wiedemann 99 parameters calibrated for Marconi Roundabout. This result indicates an acceptable level of transferability of the Wiedemann parameters between the two case studies;

(iv) in Scenario 4, the Percentage of Change related to all the significant parameters varies from a minimum of $-5.0 \%$ to a maximum of $0.6 \%$. The only parameter, which shows a high Percentage of Change value, is the Desired Speed: this percentage is a little greater than $20 \%$ which is still an acceptable value for this parameter according to [27]. These results confirm the transferability of overall parameters and corroborate the goodness of the application-based approach. 
TABLE 12: Comparison of parameters calibrated for Marconi and Cosmai roundabouts.

\begin{tabular}{|c|c|c|c|c|c|c|c|}
\hline Parameters & $\begin{array}{l}\text { Low level } \\
\qquad(-1)\end{array}$ & $\begin{array}{l}\text { High level } \\
\qquad(+1)\end{array}$ & $\begin{array}{c}\text { Default } \\
\text { VISSIM value }\end{array}$ & $\begin{array}{c}\text { Marconi } \\
\text { calibrated } \\
\text { value }\end{array}$ & $\begin{array}{c}\text { Cosmai } \\
\text { calibrated } \\
\text { value }\end{array}$ & Diff. & $\%$ of Change \\
\hline $\begin{array}{l}\text { Desired Speed } \\
\text { (average) }(\mathrm{Km} / \mathrm{h})\end{array}$ & 20 & 50 & 50 & 20.01 & 26.42 & -6.41 & -21.3 \\
\hline $\begin{array}{l}\text { Observed vehicles } \\
\text { ahead }\end{array}$ & 1 & 4 & 4 & 4 & 4 & 0 & 0 \\
\hline $\mathrm{CCO}$ & 0.5 & 3 & 1.5 & 0.50 & 0,53 & -0.03 & -1.2 \\
\hline $\mathrm{CCl}$ & 0.5 & 1.5 & 0.9 & 0.52 & 0,55 & -0.03 & -3 \\
\hline $\mathrm{CC} 2$ & 1.5 & 6 & 4 & 2,21 & 2.27 & -0.06 & -3 \\
\hline CC3 & -15 & -4 & -8 & -7.06 & $-7,15$ & -0.09 & -0.8 \\
\hline CC6 & 2 & 20 & 11.44 & 8.01 & 8.29 & -0.28 & -1.5 \\
\hline CC7 & 0.1 & 0.3 & 0.25 & 0.27 & 0.28 & -0.01 & -5 \\
\hline $\mathrm{CC} 8$ & 0.5 & 3.5 & 3.5 & 3.48 & 3.46 & 0.02 & 0.6 \\
\hline
\end{tabular}

Therefore, the results showed that, in simulation, the calibration process is fundamental to guaranteeing a correlation between observed and simulated parameters. However, an in-depth analysis is still needed to define transferability methodologies that allow applying the parameters calibrated for a case study to other similar locations.

In this paper, the authors compared data and simulation results related to only one traffic characteristic (queue length): in future studies, it will be interesting to provide evidence of the quality of the calibration results, also on the same testsite, by considering multiobjective criteria for the calibration process (average delay time, time of service, follow-up time, speed, flow, etc.). Furthermore, attention will be focused on the comparison between observed and simulated parameters for different case studies (not only for roundabouts, but, for example, for at-grade intersections, traffic-light intersections, ramps, and weaving zones).

\section{Competing Interests}

The authors declare that there is no conflict of interests regarding the publication of this paper.

\section{References}

[1] F. F. Saccomanno, F. Cunto, G. Guido, and A. Vitale, "Comparing safety at signalized intersections and roundabouts using simulated rear-end conflicts," Transportation Research Record, vol. 2078, pp. 90-95, 2008.

[2] B. B. Park and J. D. Schneeberger, "Microscopic simulation model calibration and validation, case study of VISSIM simulation model for a coordinated actuated signal system," Transportation Research Record, vol. 1856, pp. 185-192, 2003.

[3] F. G. Praticò, R. Vaiana, and V. Gallelli, "Micro-simulation effectiveness in predicting operating speed profiles in a roundabout," Advances in Transportation Studies, vol. 37, pp. 5-14, 2015.

[4] R. Vaiana, V. Gallelli, and T. Iuele, "Methodological approach for evaluation of roundabout performances through microsimulation," Applied Mechanics and Materials, vol. 253-255, no. 1, pp. 1956-1966, 2013.
[5] T. Vieira da Rocha, A. Can, C. Parzani, B. Jeanneret, R. Trigui, and L. Leclercq, "Evaluating the influence of microscopic traffic models outputs on fuel consumption estimation," Transportation Research Part D, vol. 24, pp. 17-26, 2013.

[6] TSS-Transport Simulation Systems, Aimsun (Advanced Interactive Microscopic Simulator for Urban and Non-Urban Networks), Transport Simulation Systems, Barcelona, Spain, 2012.

[7] PTV Planung Transport Verkehr. (VISSIM User Manual Version 7.0), PTV, Karlsruhe, Germany, 2014.

[8] Paramics Microsimulation, S-Paramics (Parallel Microscopic Traffic Simulator), SIAS, Edinburgh, UK, 2011.

[9] Y. H. Yap, H. M. Gibson, and B. J. Waterson, "An international review of roundabout capacity modelling," Transport Reviews, vol. 33, no. 5, pp. 593-616, 2013.

[10] G. Nikolic, R. Pringle, and K. Bragg, "Evaluation of analytical tools used for the operational analysis of roundabouts," in Proceedings of the Annual Conference of the Transportation Association of Canada, Halifax, Canada, September 2010.

[11] R. Vaiana, V. Gallelli, and T. Iuele, "Sensitivity analysis in traffic microscopic simulation model for roundabouts," The Baltic Journal of Road and Bridge Engineering, vol. 8, no. 3, pp. 174183, 2013.

[12] V. Gallelli, T. Iuele, and R. Vaiana, "Conversion of a semitwo lanes roundabout into a turbo-roundabout: a performance comparison," Procedia Computer Science, vol. 83, pp. 393-400, 2016.

[13] V. Astarita, V. Giofré, G. Guido, and A. Vitale, "Investigating road safety issues through a microsimulation model," Procedia-Social and Behavioral Sciences, vol. 20, pp. 226-235, 2011.

[14] C. Gagnon, A. W. Sadek, A. Touchette, and M. Smith, "Calibration potential of common analytical and microsimulation roundabout models: New England case study," Transportation Research Record, vol. 2071, pp. 77-86, 2008.

[15] X. Chen and M. S. Lee, "A case study on multi-lane roundabouts under congestion: comparing software capacity and delay estimates with field data," Journal of Traffic and Transportation Engineering, vol. 3, no. 2, pp. 154-165, 2016.

[16] I. Ištoka Otković, T. Tollazzi, and M. Šraml, "Calibration of microsimulation traffic model using neural network approach," Expert Systems with Applications, vol. 40, no. 15, pp. 5965-5974, 2013. 
[17] R. Arroju, H. K. Gaddam, L. D. Vanumu, and K. Ramachandra Rao, "Comparative evaluation of roundabout capacities under heterogeneous traffic conditions," Journal of Modern Transportation, vol. 23, no. 4, pp. 310-324, 2015.

[18] J. Barceló, Fundamentals of Traffic Simulation, Springer, Berlin, Germany, 2010.

[19] M. Essa and T. Sayed, "Transferability of calibrated microsimulation model parameters for safety assessment using simulated conflicts," Accident Analysis and Prevention, vol. 84, pp. 41-53, 2015.

[20] B. Ciuffo, J. Casas, M. Montanino, J. Perarnau, and V. Punzo, "Gaussian process metamodels for sensitivity analysis of traffic simulation models. Case Study of AIMSUN Mesoscopic Model," Transportation Research Record, vol. 2390, pp. 87-98, 2013.

[21] T. Tettamanti, A. Csikós, I. Varga, and A. Eleőd, "Iterative calibration of VISSIM simulator based on genetic algorithm," Acta Technica Jaurinensis, vol. 8, no. 2, pp. 145-152, 2015.

[22] B. Ciuffo, V. Punzo, and V. Torrieri, "Comparison of simulationbased and model-based calibrations of traffic-flow microsimulation models," Transportation Research Record, vol. 2088, no. 1, pp. 36-44, 2008.

[23] European Commission-Joint Research Centre, "Traffic Simulation: Case for guidelines. MULTITUDE Project-COST Action TU0903," 2016, http://publications.jrc.ec.europa.eu/repository/ handle/JRC88526.

[24] Ministero delle infrastrutture e dei Trasporti, "Decreto Ministeriale 19/04/2006-Norme funzionali e geometriche per la costruzione delle intersezioni stradali”.

[25] G. Guido, F. F. Saccomanno, A. Vitale, V. Astarita, and D. C. Festa, "Comparing safety performance measures obtained from video capture data," Journal of Transportation Engineering, vol. 137, no. 7, pp. 481-492, 2011.

[26] R. Wiedemann, Simulation des Strassenverkehrsflusses, Schriftenreihe des Instituts für Verkehrswesen der Universität Karlsruhe, Band 8, Karlsruhe, Germany, 1974.

[27] F. G. Praticò, R. Vaiana, and V. Gallelli, “Transport and traffic management by micro simulation models: operational use and performance of roundabouts," in Proceedings of the 18th International Conference on Urban Transport and the Environment (UT '12), pp. 383-394, Coruña, Spain, May 2012.

[28] F. Cunto and F. F. Saccomanno, "Calibration and validation of simulated vehicle safety performance at signalized intersections," Accident Analysis and Prevention, vol. 40, no. 3, pp. 11711179, 2008.

[29] G. Guido, F. F. Saccomanno, A. Vitale, V. Gallelli, and D. Rogano, "A calibration framework of car following models for safety analysis based on vehicle tracking data from smartphone probes," International Journal of Mobile Network Design and Innovation, vol. 5, no. 4, pp. 205-212, 2014.

[30] N. E. Lownes and R. B. Machemehl, "Sensitivity of simulated capacity to modification of VISSIM driver behavior parameters," Transportation Research Record, vol. 1988, pp. 102-110, 2006.

[31] D. C. Montgomery, Design and Analysis of Experiments, John Wiley \& Sons, Hoboken, NJ, USA, 6th edition, 2005.

[32] European Commission-Joint Research Centre. The Calibration of Traffic Simulation Models. Report on the assessment of different Goodness of Fit measures and Optimization Algorithms MULTITUDE Project-COST Action TU0903, http:// publications.jrc.ec.europa.eu/repository/handle/JRC68403.
[33] D. K. Hale, C. Antoniou, M. Brackstone, D. Michalaka, A. T Moreno, and K. Parikh, "Optimization-based assisted calibration of traffic simulation models," Transportation Research Part C: Emerging Technologies, vol. 55, pp. 100-115, 2015.

[34] S. Sikder and A. Pinjari, "Spatial transferability of person-level daily activity generation and time use models: an Empirical Assessment," Transportation Research Record: Journal of the Transportation Research Board, vol. 2343, pp. 95-104, 2013.

[35] S. Sikder, B. Augustin, A. R. Pinjari, and N. Eluru, "Spatial transferability of tour-based time-of-day choice models," Transportation Research Record, vol. 2429, pp. 99-109, 2014. 


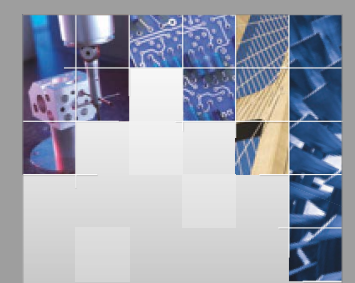

\section{Enfincering}
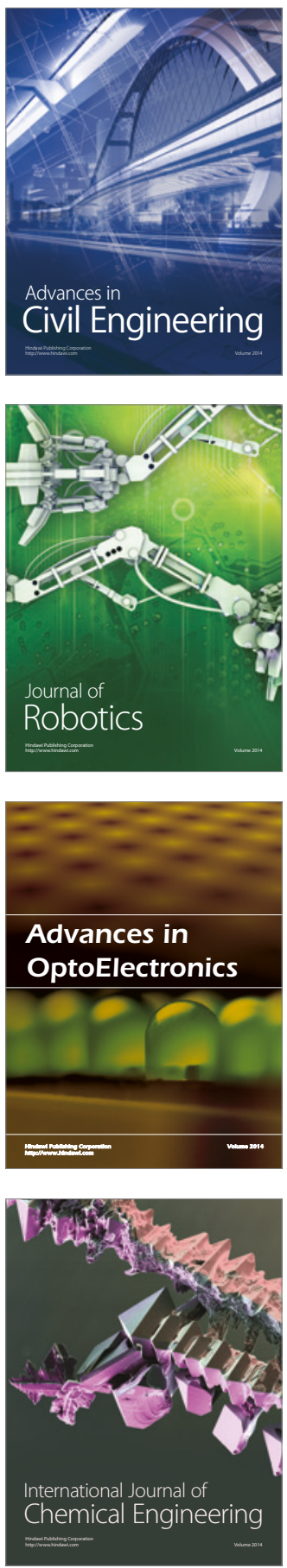

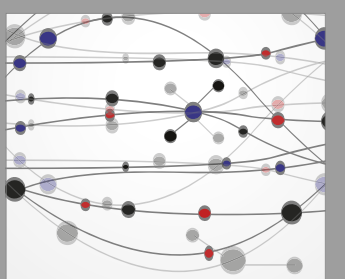

The Scientific World Journal

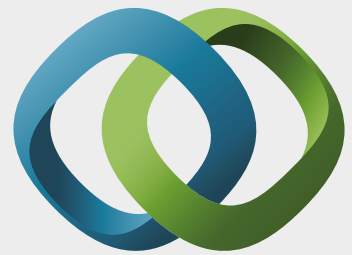

\section{Hindawi}

Submit your manuscripts at

https://www.hindawi.com
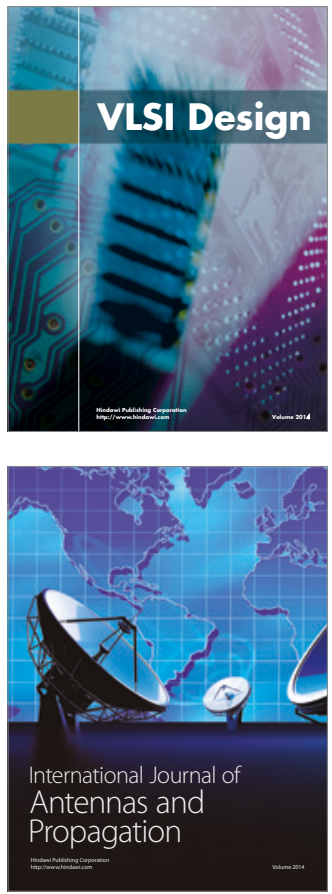

\section{Rotating}

Machinery
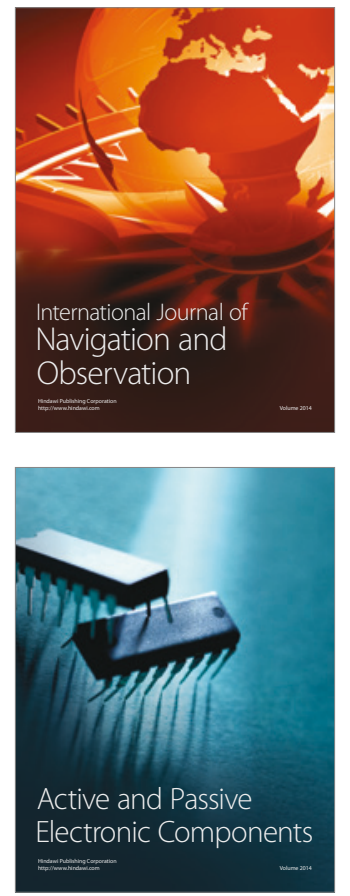
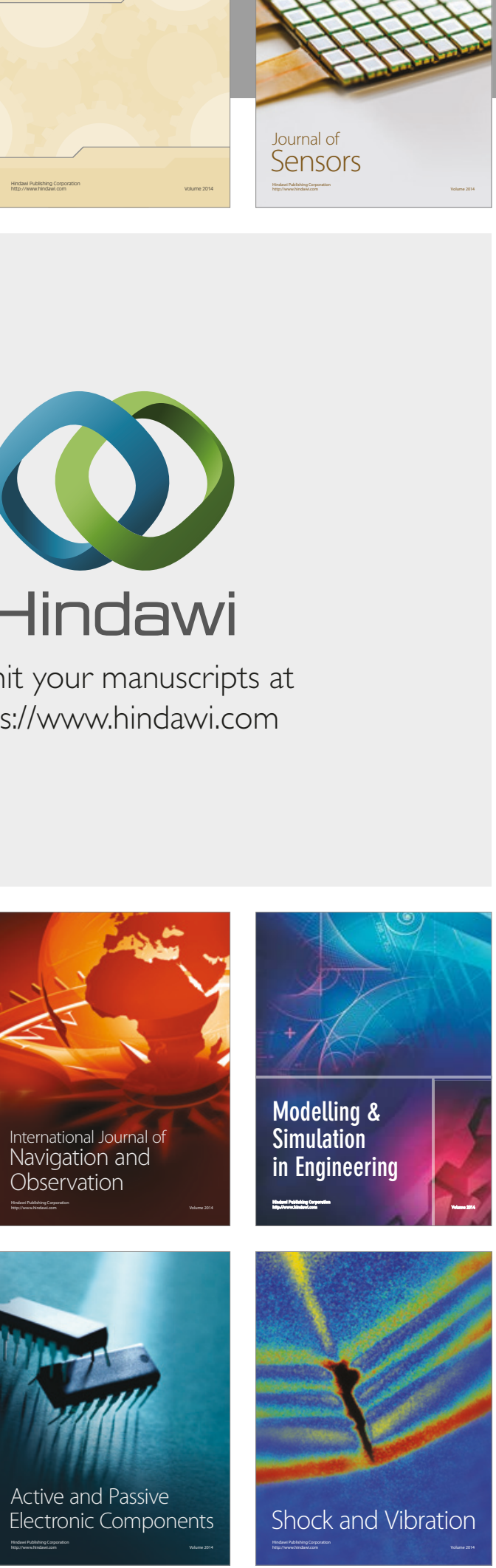
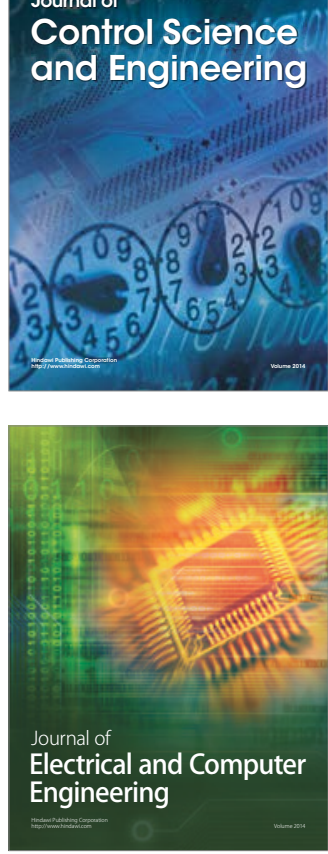

Distributed

Journal of

Control Science

and Engineering
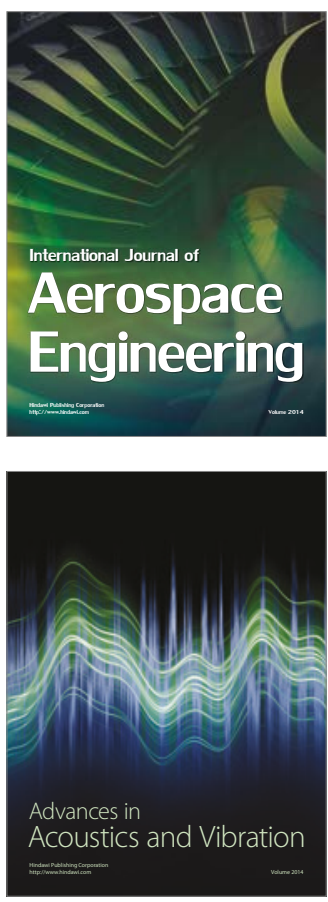

Sensor Networks 